Article

\title{
General Pathway for a Convenient One-Pot Synthesis of Trifluoromethyl-Containing 2-amino-7-alkyl(aryl/heteroaryl)- 1,8-naphthyridines and Fused Cycloalkane Analogues
}

\author{
Helio G. Bonacorso *, Rosália Andrighetto, Nícolas Krüger, Nilo Zanatta and \\ Marcos A. P. Martins
}

Núcleo de Química de Heterociclos (NUQUIMHE), Departamento de Química, Universidade Federal de Santa Maria, 97105-900 Santa Maria, RS, Brazil

* Author to whom correspondence should be addressed; E-Mail: heliogb@base.ufsm.br;

Tel.: +55(55)-3220-8867; Fax: +55(55)-3220-8031.

Received: 15 February 2011; in revised form: 24 March 2011 / Accepted: 28 March 2011 /

Published: 30 March 2011

\begin{abstract}
A convenient and general method for the synthesis in $26-73 \%$ yields of a new series of 7-alkyl(aryl/heteroaryl)-2-amino-5-trifluoromethyl-1,8-naphthyridines from direct cyclocondensation reactions of 4-alkoxy-1,1,1-trifluoroalk-3-en-2-ones $\left[\mathrm{CF}_{3} \mathrm{C}(\mathrm{O}) \mathrm{CH}=\mathrm{C}\left(\mathrm{R}^{1}\right) \mathrm{OR}\right.$, where $\mathrm{R}^{1}=\mathrm{H}, \mathrm{Me}, \mathrm{Ph}, 4-\mathrm{MePh}, 4-\mathrm{OMePh}, 4-\mathrm{FPh}, 4-\mathrm{BrPh}, 4-\mathrm{NO}_{2} \mathrm{Ph}$, 2-furyl, 2-thienyl and $\mathrm{R}=\mathrm{Me}$, Et] with 2,6-diaminopyridine (2,6-DAP), under mild conditions, is described. Another synthetic route also allowed the synthesis of 2-amino-5-trifluoromethylcycloalka[b][1,8]naphthyridines in $33-36 \%$ yields, from direct or indirect cyclocondensation reactions of five-, six- and seven-membered 2-trifluoroacetyl-1-methoxycycloalkenes with 2,6-DAP.
\end{abstract}

Keywords: trifluoromethylated heterocyles; quinolines; naphthyridines; cycloalka[b][1,8]naphthyridines; aminonaphthyridines

\section{Introduction}

Among the nitrogenous heterocycles, naphthyridines and their derivatives represent an important class of organic molecules that attract the interest of both synthetic and medicinal chemists due to their exceptionally broad spectrum of biological activities as well as their use as important binding units in 
the molecular design of synthetic receptors [1]. Naphthyridine derivatives have attracted considerable attention primarily due to the presence of a 1,8-naphthyridine skeleton in many compounds which have been isolated from natural substances and exhibit various biological activities [2]. As a heterocyclic moiety, 1,8-naphthyridine also deserves special interest as in its molecule, the arrangement of the nitrogen atoms is optimal for chelation of various metal cations, including lanthanide ions [3].

In parallel to the growing interest in the synthesis of 1,8-naphthyridines to provide biologically active molecules, a large number of publications have reported that several of their derivatives possess antibacterial [4], antimycobacterial [5], antitumor [6], anti-inflammatory [7,8], analgesic [8], antiplatelet [9], gastric antisecretary [10], local anaesthetic [11], anticonvulsant [12] and antihypertensive activity [13,14], besides being associated with $\beta$-adrenergic blocking properties [15]. Some 1,8-naphthyridine compounds have been patented as fungicides, bactericides, insecticides, herbicides, anxiolytic, antihypertensives, antiarrhythmics and also as immunostimulants [2,16-19].

In addition, has been recognized that attachment of a trifluoromethyl group into heterocycles can be used to modulate the physical, chemical and biological properties. It is well documented that the influence of the trifluoromethyl substituent on physiological activity is due mainly to the increased lipophilicity of the molecules, causing greater cell permeability and resistance to enzyme degradation [20]. Consequently, synthetic methodology to incorporate fluorine and fluorous synthons must be improved in order to prepare sophisticated fluoroorganic molecules on a practical scale. One of the most satisfactory methods for introducing a $\mathrm{CF}_{3}$ group into heterocycles is via the trifluoromethylated building block approach. The trifluoroacetylation of enol ethers or acetals provided, in one step and in good yields, $\beta$-alkoxyvinyl trifluoromethyl ketones 1 which proved to be useful building blocks for the syntheses of many series of heterocyclic compounds [21].

Since the 50s various diamino-ketoester condensations involving reactions of cyclic and acyclic $\beta$ ketoesters or diketones with aminopyridines or diaminopyridines have been studied in an attempt to develop generalized predictions regarding the direction of ring closure to form diazepinones, naphthyridones, naphthyridines or pyrimidines $[6,12-15,19,22]$. Whereas a literature review shows that the synthesis of trifluoromethylated naphthyridines and derivatives has been little explored and that 1,8-naphthyridines trifluoromethylated described are associated with satisfactory biological activities [14], the incorporation of trifluoromethyl group in a variety of 1,8-naphthyridines would be expected to provide highly desirable intermediates for the synthesis of new drug candidates. So, due to the great biological importance and employment of amino-naphthyridines as starting material for the synthesis of new tri and tetracyclic heterocycles, the development of new synthetic approaches remains an active research area $[19,23]$.

The use of diethyl ethoxymethylenemalonate (EMME) [24], Conrad-Limpach [25], Knorr [26] and Skraup [27] methods have been particularly successful in the synthesis of certain quinolines. The adaptation of these reactions to the synthesis of the corresponding naphthyridines by employing aminopyridines instead of anilines should furnish convenient methods for the preparation of these types of compounds since aminopyridines are readily available [19]. However, these methods have not been as satisfactory for the preparation of 1,8-naphthyridines as they are the preparation of quinolines. In contrast to aniline derivatives, 2-aminopyridine derivatives may cyclize in two ways, one of which leads to the formation of 1,8-naphthyridines and the other leads to the formation of pyrimidines, and the latter course of reaction has is observed with more frequency [28]. In both types of cyclization, the 
pyridine ring functions as the electron donor and the carbonyl group in the side chain serves as the electron acceptor.

The formation of pyrimidines should not be surprising, since the resonance structures existing in the structure of the 2-aminopyridine derivatives strongly favor cyclization leading to the pyrimidine ring. Nevertheless, although the formation of a pyrimidine often occurs, an investigation of the synthesis of certain 1,8-naphthyridines from aminopyridines with diethylmalonate, ethoxymethylidenemalonate or ethyl acetoacetate has been made [14,19,29]. It is known that the synthesis of 1,8-naphthyridines has been performed successfully when 6-methyl-2-aminopyridine or 2,6-diaminopyridine are used as precursors, since 6-methyl or 6-amino groups activate the 3-position leading to those molecules $[14,29]$. Thus, the great difference in behavior of 2-aminopyridine and 2,6-diaminopyridine, for example, has usually been attributed to activation of the 3-position by the electron releasing amine group.

Recently, we reported reactivity of the endocyclic nitrogen atom of the $\pi$-deficient pyridine ring towards the carbonyl group of the trichloroacetyl enamine derivates from the reactions employing 4alkoxy-4-alkyl(aryl)-1,1,1-trichloroalk-3-en-2-ones and 2-aminopyridine in a molar ratio of 1:1, presenting a convenient method to obtain 4-oxo-4H-pyrido[1,2-a]pyrimidines in good yields (45-81\%) [28]. We also reported the synthesis of $5 H$-thiazolo[3,2-a]pyrimidi-5-ones from the reactions of $\beta$ alkoxyvinyl trichloromethyl ketones and 2-aminothiazole [30]. Unfortunately, reactions using $\beta$ alkoxyvinyl trifluoromethyl ketones and 2-aminopyridine in an attempt to obtain the respective cyclic structure only resulted in the isolation of trifluoroacetylenamine derivatives [32]. On the other hand, reactions using $\beta$-alkoxyvinyl trifluoro(chloro)methyl ketones and 2,3-diaminopyridine have been successfully employed in the synthesis of $3 H$-pyrido[2,3-b][1,4]diazepinols [31,32] or diazepinones [31,33].

Although the reactions of 4-alkoxy-4-alkyl(aryl/heteroaryl)-1,1,1-trifluoroalk-3-en-2-ones with primary and secondary amines have been well documented [28,30-41], there are no reports in the literature dealing with $\beta$-alkoxyvinyl trifluoromethyl ketones as electrophilic precursors and 2,6diaminopyridine (2,6-DAP) as nucleophilic precursor. Considering the importance of trifluoromethylated heterocycles, as an extension of our research the purpose of this paper is to report the results of a chemical behavior study of the reactions of 4-alkoxy-4-alkyl(aryl/heteroaryl)-1,1,1trifluoroalk-3-en-2-ones and 2-trifluoroacetyl-1-methoxycycloalkenes 1 with 2,6-DAP, a symmetrical heteroaromatic diamine, aiming at the synthesis of new nitrogen-containing trifluoromethylated heterocycles with conventional procedures (Scheme 1). None of methods reported to date for the synthesis of naphthyridines employs the strategy adopted in this study. Our method allows the easier introduction of $\mathrm{CF}_{3}$ group at position 5 and of wide scope of both electron-donor and electronwithdrawing substituents at position 7 and fused cycloalkanes to the C6-C7 bond of the naphthyridines ring. Furthermore, the free amino group at position 2, in both cases, allows further important derivatizations.

\section{Results and Discussion}

Initially, a series of ten examples of 4-alkoxy-4-alkyl(aryl/heteroaryl)-1,1,1-trifluoroalk-3-en-2-ones $\mathbf{1 a - j}$, which are readily available $C C C$ synthetic blocks, were prepared from trifluoroacetylation 
reactions of enol ethers commercially available (for 1a-b) or generated in situ from the respective acetophenone dimethyl acetal (for 1c-j) with trifluoroacetic anhydride, respectively, in the presence of pyridine, as described in the literature [42-45].

Fortunately, we found that trifluoromethylated ketones 1a-j when added dropwise to 2,6-DAP at a molar ratio of $1: 1$ in methanol as solvent at $0{ }^{\circ} \mathrm{C}$ for 2 hours and after heating under reflux for 24 hours, produced 7-alkyl(aryl/heteroaryl)-2-amino-5-trifluoromethyl-1,8-naphthyridines 3a-j. These compounds were easily isolated from the one-step reaction mixtures in 51-73\% yields, although $\mathbf{3 a}, \mathbf{3 b}$ and 3i were obtained in lower yields 26, 39 and 38\%, respectively (Scheme 1). This is not surprising since a detailed review of the literature shows that enaminone derivatives of $\beta$-ethoxyvinyl trifluoromethyl ketone (enone 1a) present a different chemical behavior from other enones [41], leading to heterocycles with lower yields [34] and the absence of cyclization has been reported in many papers $[28,35]$. The naphthyridine $\mathbf{3 b}$ was previously also synthesized in a low yield (10-23\%) by Eichler et al. from the reaction of 2,6-DAP and 1,1,1-trifluoropentane-2,4-dione [22].

As an extension of this study we also developed the synthesis of compound $\mathbf{3 b}$ from the cyclocondensation reaction of enamino ketone intermediate $\mathbf{2 b}$ in methanol at reflux temperature for 24 hours (Scheme 1). The isolation of $\mathbf{2} \mathbf{b}$ was possible when the reaction of enone $\mathbf{1 b}$ with 2,6-DAP was carried out in methanol as solvent at $0{ }^{\circ} \mathrm{C}$ for 2 hours. Unfortunately, the enamino ketones $\mathbf{2 a}, \mathbf{2 c - j}$ could be not isolated as pure compounds, under the same or similar reaction conditions.

Scheme 1. Synthesis of 7-alkyl(aryl/heteroaryl)-2-amino-5-trifluoromethyl-1,8-naphthyridines.

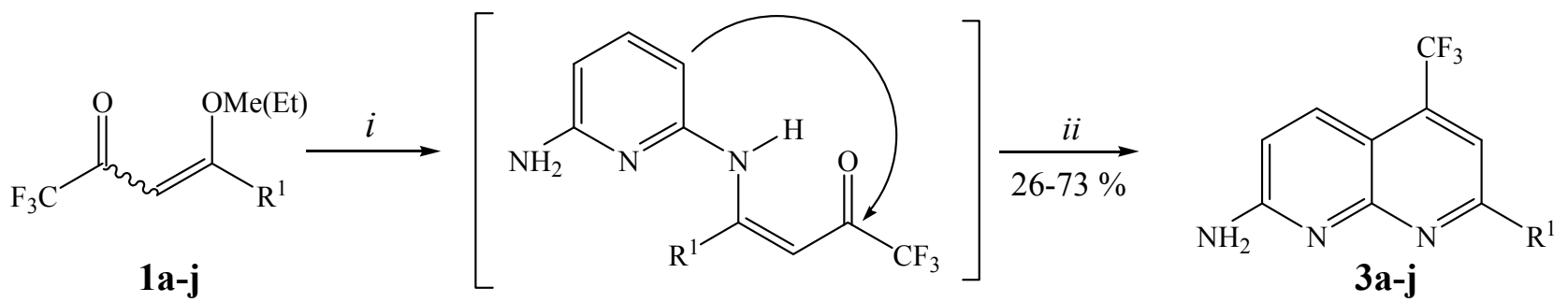

2a, 2c-j (not isolated)

2b (isolated, $58 \%$ )

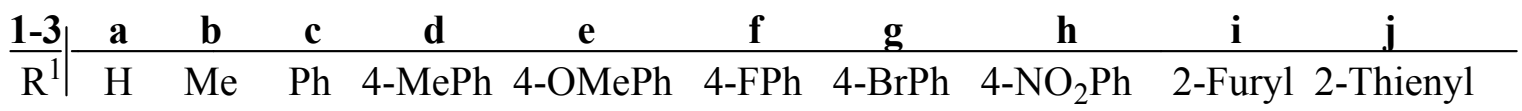

Reagents and Conditions: $(i)=2,6$-Diaminopyridine, $\mathrm{MeOH}, 0{ }^{\circ} \mathrm{C}, 2 \mathrm{~h}$; (ii) $=\mathrm{MeOH}$, reflux, $24 \mathrm{~h}$.

The structures of 3a-j were established on the basis of ${ }^{1} \mathrm{H}$ - and ${ }^{13} \mathrm{C}-\mathrm{NMR}$ spectroscopy and literature data for similar compounds (chemical shifts and spin \pm spin coupling constants) $[5,9,12,14,22,41,46]$. According to the literature, it is well known that the proton in the 4-position of the naphthyridine nucleus shows long-range coupling with fluorine atoms of the 5-trifluoromethyl substituent, but in some cases the outer signals of the quartets can appear as shoulders on the inner signals instead of as clearly resolved quartets [22]. This splitting of the $\mathrm{H}-4$ signal is seen in all of the compounds having this structural feature and was clearly seen in ${ }^{1} \mathrm{H}-\mathrm{NMR}$ spectral data of compounds 3a-j. 
Furthermore, some examples of compounds 3 were converted in good yields (82-93\%) to the corresponding 2-acetamide derivatives 4 , by reaction with acetic anhydride under high temperature [14] (Scheme 2).

Scheme 2. Synthesis of 2-acetamide derivatives.<smiles>[R]c1cc(C(F)(F)F)c2ccc(N)nc2n1</smiles>

3

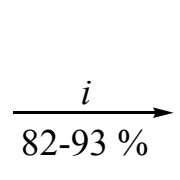

$\mathrm{H}_{3} \mathrm{C}^{\prime}$<smiles>C</smiles><smiles>C1CCCCC1</smiles><smiles>[R]c1cc(C(F)(F)F)c2ccc(N)nc2n1</smiles>

4

$$
\begin{array}{c|ccccc}
\mathbf{3 , 4} & \mathbf{c} & \mathbf{f} & \mathbf{g} & \mathbf{i} & \mathbf{j} \\
\hline \mathrm{R}^{1} & \mathrm{Ph} & \text { 4-FPh } & \text { 4-BrPh } & \text { 2-Furyl } & \text { 2-Thienyl }
\end{array}
$$

Reagents and Conditions: $(i)=$ Acetic anhydride, $160^{\circ} \mathrm{C}, 4 \mathrm{~h}$.

As a second extension, applying a similar cyclization method to that previously described by us for the preparation of some trifluoromethyl-substituted benzo[ $h]$ quinolines [35], dihydrobenzo[c]acridines [36], cycloalka[b]quinolines [37], 1,2,3,4-tetrahydroacridines [38], 7-aminoquinolines and 1,7phenanthrolines [41], to investigate the chemical behavior of enamino ketone intermediate $\mathbf{2 b}$ in polyphosphoric acid medium (PPA), we found that a 1:1 mixture of isomers $\mathbf{3 b} \mathbf{5} \mathbf{5} \mathbf{b}$ is obtained when 2b is heated at $90{ }^{\circ} \mathrm{C}$ for $20 \mathrm{~h}$ (Scheme 3). These compounds were easily identified since $\mathrm{CH}_{3}$ and $\mathrm{CF}_{3}$ groups have different chemical shifts in the ${ }^{13} \mathrm{C}-\mathrm{NMR}$ spectra of each of the isomers. Thus, 7-methyl2-amino-5-trifluoromethyl-1,8-naphthyridine (3b) showed chemical shifts at $133.8 \mathrm{ppm}\left(\mathrm{C} 5, \mathrm{q},{ }^{2} J_{C F} 31\right.$ $\mathrm{Hz}$ ) and $24.6 \mathrm{ppm}$ for the $\mathrm{CH}_{3}$ group, while the isomer 5-methyl-2-amino-7-trifluoromethyl-1,8naphthyridine (5b) showed chemical shifts at $148.1 \mathrm{ppm}\left(\mathrm{C} 7, \mathrm{q},{ }^{2} J_{C F} 34 \mathrm{~Hz}\right)$ and $17.5 \mathrm{ppm}$ for the $\mathrm{CH}_{3}$ group.

Scheme 3. Synthesis of isomers 3b:5b.<smiles>C/C(=C/C(=O)C(F)(F)F)Nc1cccc(N)n1</smiles>

2b<smiles>Cc1cc(C(F)(F)F)c2ccc(N)nc2n1</smiles>

$3 \mathbf{b}(44 \%)$<smiles>Cc1cc(C(F)(F)F)nc2nc(N)ccc12</smiles>

$5 \mathbf{b}(43 \%)$

Reagents and Conditions: $(i)=\mathrm{PPA}, 90^{\circ} \mathrm{C}, 20 \mathrm{~h}$.

As an second extension of this study, we also developed the synthesis of trifluoromethyl substituted cycloalka[b][1,8]naphthyridines $\mathbf{3}$ from the reactions of 2-trifluoroacetyl-1-methoxycycloalkenes $\mathbf{1}$ with 2,6-DAP (Scheme 4). Firstly, three examples of methoxycycloalkenes $\mathbf{1 k - m}$ were obtained by a direct acylation reaction of the cycloalkane dimethyl acetals with trifluoracetic anhydride in the presence of pyridine, as described in the literature [47,48]. Subsequently, the intramolecular cyclization reactions of trifluoroacetylated cycloalkenes $\mathbf{1 k - m}$ were carried out, applying the same 
conditions described for the preparation of 3a-j. This reaction condition allows to isolate, in one-pot, only the respective cycloalka[b][1,8]naphthyridines $\mathbf{3 1}, \mathbf{3 m}$ in $30-33 \%$ yields because the cyclization of $\mathbf{1 k}$ did not take place. This reaction condition allowed the isolation of enaminone $\mathbf{2 k}$ in $43 \%$ yield, derived from 2-trifluoroacetyl-1-methoxycyclopentene. The synthesis of $\mathbf{3 k}$, in $78 \%$ yield, was only possible from intramolecular cyclization reaction of enaminone $\mathbf{2 k}$ in polyphosphoric acid medium (PPA), as shown in Scheme 4. The structures of compounds $\mathbf{2 k}, \mathbf{3 k}-\mathbf{m}$ were easily established on the basis of ${ }^{1} \mathrm{H}$ - and ${ }^{13} \mathrm{C}-\mathrm{NMR}$ spectroscopy and literature data for similar compounds, such as trifluoromethyl-containing cycloalka[b]quinolines [37].

Scheme 4. Synthesis of trifluoromethyl substituted cycloalka[b][1,8]naphthyridines.

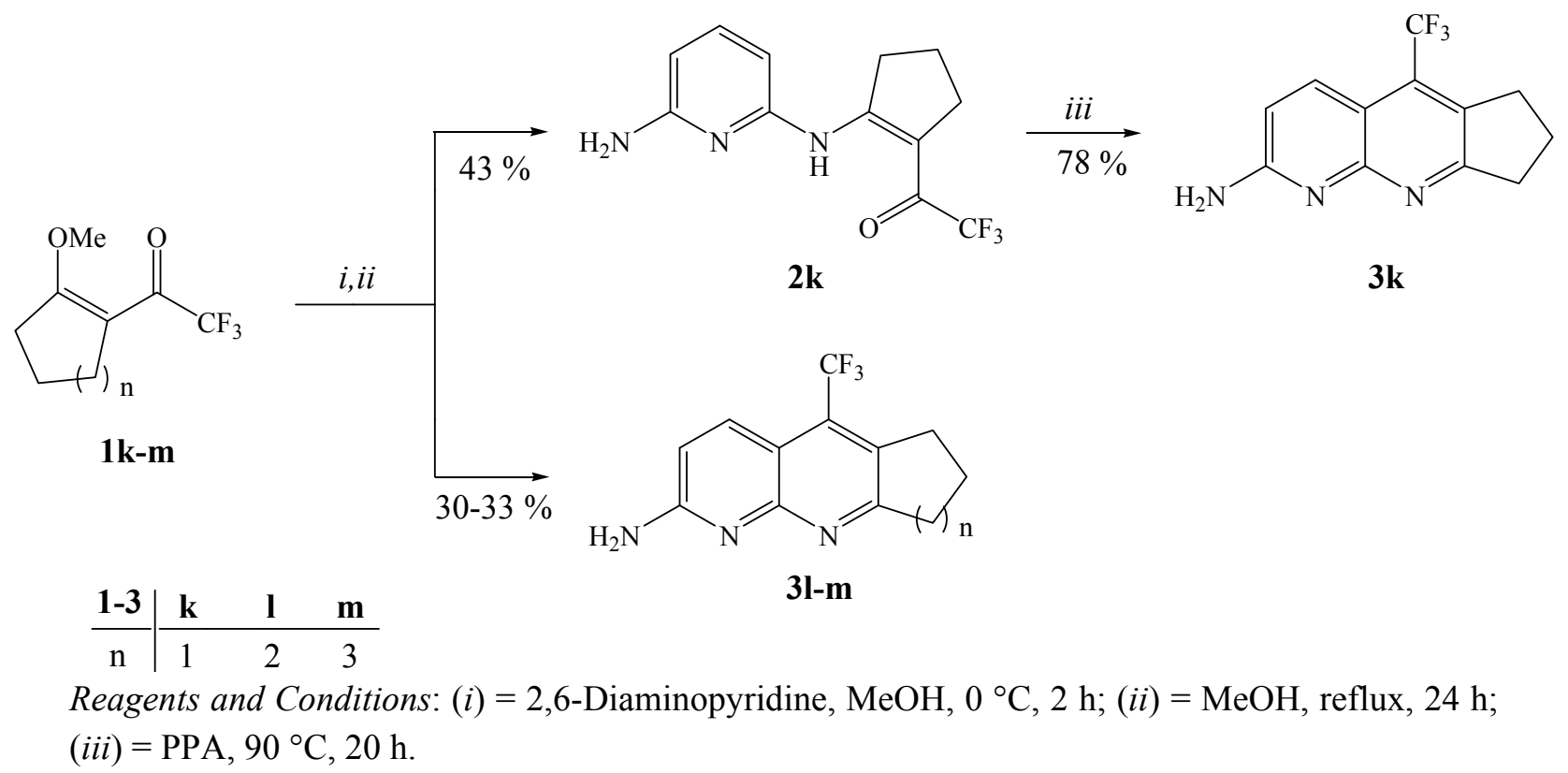

Any attempt to explain the low yield for the synthesis of compounds $\mathbf{3 I}$ and $\mathbf{3 m}$ and the absence of cyclization for $\mathbf{1 k}$ requires an examination of the structural effects on internal cycloalkenes $\mathbf{1 k}-\mathbf{m}$. Information from the literature for precursors of these compounds [47], obtained by calculation using AM1 semi-empirical method, indicate the possibility of non-planar conformations for these molecules, which would explain the difficulty in achieving [3+3] intramolecular cyclization in the case of $1 \mathbf{k}[37,48]$.

\section{Experimental}

\subsection{General}

Unless otherwise indicated all common reagents and solvents were used as obtained from commercial suppliers without further purification. The melting points were determined using a Kofler Reichert-Thermovar and Electrothermal Mel-Temp 3.0 apparatus. ${ }^{1} \mathrm{H}$ - and ${ }^{13} \mathrm{C}-\mathrm{NMR}$ spectra were acquired on a Bruker DPX 200 spectrometer $\left({ }^{1} \mathrm{H}\right.$ at $\left.200.13 \mathrm{MHz}\right)$ and Bruker DPX $400\left({ }^{1} \mathrm{H}\right.$ at $400.13 \mathrm{MHz},{ }^{13} \mathrm{C}$ at $100.32 \mathrm{MHz}$ ) spectrometer, $5 \mathrm{~mm}$ sample tubes, $298 \mathrm{~K}$, digital resolution \pm 0.01 ppm, in DMSO- $d_{6}$, in $\mathrm{CDCl}_{3}$ for $\mathbf{3 a}, \mathbf{3 m}, \mathbf{4 i}, \mathbf{4} \mathbf{j}$ and $\mathrm{CDCl}_{3}+\mathrm{TFA}$ for $\mathbf{4 g}$, using TMS as internal 
reference. Mass spectra were registered in a HP 6890 GC connected to a HP 5973 MSD and interfaced by a Pentium PC. The GC was equipped with a split-splitless injector, autosampler, cross-linked HP-5 capillary column (30 m, $0.32 \mathrm{~mm}$ of internal diameter), and helium was used as the carrier gas. The CHN elemental analyses were performed on a Perkin-Elmer $2400 \mathrm{CHN}$ elemental analyzer (São Paulo University, USP/Brazil).

\subsection{General procedure for the synthesis of (Z)-N(5,5,5-Trifluoro-4-oxo-2-penten-2-yl)-2,6-diamino-} pyridine (2b)

To a magnetically stirred solution of 2,6-diaminopyridine $(0.54 \mathrm{~g}, 5 \mathrm{mmol})$ in methanol $(25 \mathrm{~mL})$, a solution of $1 \mathbf{b}(0.84 \mathrm{~g}, 5 \mathrm{mmol})$ in methanol $(25 \mathrm{~mL})$ was added dropwise at $0{ }^{\circ} \mathrm{C}$ over a period of $2 \mathrm{~h}$. After the end of the reaction, the solvent was evaporated under reduced pressure. Then, the crude oily product was dissolved in hot ethanol and subsequently cooled $\left(4-8{ }^{\circ} \mathrm{C}, 24 \mathrm{~h}\right)$ to give the title compound $2 \mathbf{b}(0.28 \mathrm{~g}, 58 \%$ yield $)$ as a brown solid, m.p. $130-132{ }^{\circ} \mathrm{C} .{ }^{1} \mathrm{H}-\mathrm{NMR}\left(200 \mathrm{MHz}, \mathrm{DMSO}-d_{6}\right)$ : $\delta=12.69$ (s, 1H, NH), 7.40 (t, $J=8 \mathrm{~Hz}, 1 \mathrm{H}, \mathrm{H}-10), 6.33$ (d, $J=8 \mathrm{~Hz}, 1 \mathrm{H}, \mathrm{H}-9), 6.34$ (d, $J=8 \mathrm{~Hz}, 1 \mathrm{H}$, $\mathrm{H}-11), 5.49(\mathrm{~s}, 1 \mathrm{H}, \mathrm{H}-3), 5.20\left(\mathrm{~s}, 2 \mathrm{H}, \mathrm{NH}_{2}\right), 2.53\left(\mathrm{~s}, 3 \mathrm{H}, \mathrm{CH}_{3}\right) .{ }^{13} \mathrm{C}-\mathrm{NMR}\left(100 \mathrm{MHz}, \mathrm{DMSO}-d_{6}\right): \delta=$ $175.3\left(\mathrm{q},{ }^{2} J=31 \mathrm{~Hz}, \mathrm{C}=\mathrm{O}\right), 166.5$ (C-2), 157.7 (C-8), $149.0(\mathrm{C}-6), 138.7$ (C-10), 116.6 (q, ${ }^{1} J=288 \mathrm{~Hz}$, $\left.\mathrm{CF}_{3}\right), 104.5,103.8$ (C-9, C-11), 91.8 (C-3), $21.7\left(\mathrm{CH}_{3}\right)$. GC-MS (EI, $\left.70 \mathrm{eV}\right) \mathrm{m} / z: 245\left(\mathrm{M}^{+}, 100\right), 228$ (15), 176 (22), 148 (15), 69 (5\%). Anal. Calcd. For $\mathrm{C}_{10} \mathrm{H}_{10} \mathrm{~F}_{3} \mathrm{~N}_{3} \mathrm{O}$ (245.08): C, 48.98; H, 4.11; N, 17.14\%. Found: C, 48.82; H, 4.03; N, 17.28\%.

\subsection{General procedure for the synthesis of 2-(6-Aminopyridin-2-ylamino)1-trifluoroacetyl-cyclopent-} 1-ene (2k)

To a magnetically stirred solution of 2,6-diaminopyridine $(0.22 \mathrm{~g}, 2 \mathrm{mmol})$ in methanol $(20 \mathrm{~mL})$, a solution of $1 \mathbf{k}(0.38 \mathrm{~g}, 2 \mathrm{mmol})$ in methanol $(20 \mathrm{~mL})$ was added dropwise at $0{ }^{\circ} \mathrm{C}$ over a period of $2 \mathrm{~h}$. The mixture was refluxed for an additional $24 \mathrm{~h}$. After the end of the reaction, the solvent was evaporated under reduced pressure. Then, the crude oily product was dissolved in hot ethanol and subsequently cooled $\left(4-8{ }^{\circ} \mathrm{C}, 24 \mathrm{~h}\right)$ to give the title compound $2 \mathbf{k}(0.22 \mathrm{~g}, 43 \%$ yield $)$ as a brown solid,

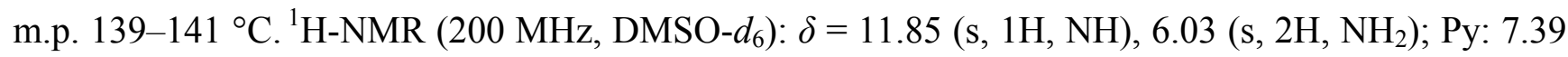
(t $J=8 \mathrm{~Hz}, 1 \mathrm{H}, \mathrm{H}-4), 6.32$ (d, $J=8 \mathrm{~Hz}, 1 \mathrm{H}, \mathrm{H}-5), 6.27$ (d, $J=8 \mathrm{~Hz}, 1 \mathrm{H}, \mathrm{H}-3)$; $c$-Pent: 3.28-3.24 (m, $\left.2 \mathrm{H}, \mathrm{CH}_{2}\right), 2.67-2.64\left(\mathrm{~m}, 2 \mathrm{H}, \mathrm{CH}_{2}\right), 1.96-1.88\left(\mathrm{~m}, 2 \mathrm{H}, \mathrm{CH}_{2}\right) .{ }^{13} \mathrm{C}-\mathrm{NMR}\left(100 \mathrm{MHz}, \mathrm{DMSO}-d_{6}\right)$ : $\delta=172.5\left(\mathrm{q},{ }^{2} J=33 \mathrm{~Hz}, \mathrm{C}=\mathrm{O}\right), 171.3(\mathrm{C}-2), 101.0(\mathrm{C}-1), 117.2\left(\mathrm{q},{ }^{1} J=289 \mathrm{~Hz}, \mathrm{CF}_{3}\right) ;$ Py: $158.8(\mathrm{C}-6)$, 149.6 (C-2), 139.0 (C-4), 104.1, 103.5 (C-3, C-5); c-Pent: $34.1\left(\mathrm{CH}_{2}\right), 26.3$ (q, $\left.{ }^{4} \mathrm{~J}=3 \mathrm{~Hz}, \mathrm{CH}_{2}\right), 22.1$ $\left(\mathrm{CH}_{2}\right)$. GC-MS (EI, $\left.70 \mathrm{eV}\right) \mathrm{m} / z$ : 271 (50), 256 (5), 202 (25), $174\left(\mathrm{M}^{+}, 100 \%\right)$. Anal. Calcd. For $\mathrm{C}_{12} \mathrm{H}_{12} \mathrm{~F}_{3} \mathrm{~N}_{3} \mathrm{O}$ (271.09): C, 53.14; H, 4.46; N, 15.49\%. Found: C, 53.02; H, 4.38; N, 15.61\%.

\subsection{General procedure for the synthesis of 7-alkyl(aryl/heteroaryl)-2-amino-5-trifluoromethyl-1,8-} naphthyridines 3a-j

To a magnetically stirred solution of 2,6-diaminopyridine (1.08 g, $10 \mathrm{mmol})$ in methanol (40 $\mathrm{mL})$, a solution of 1a-j $(10 \mathrm{mmol})$ in methanol $(40 \mathrm{~mL})$ was added dropwise at $0{ }^{\circ} \mathrm{C}$ over a period of $2 \mathrm{~h}$. The mixture was refluxed for an additional $24 \mathrm{~h}$. After the end of the reaction, the solvent was evaporated 
under reduced pressure. The crude product was dissolved in ethanol and cooled $\left(4-8{ }^{\circ} \mathrm{C}, 24 \mathrm{~h}\right)$. The solids $3 \mathbf{b}$-j were isolated from the cooled solution by filtration under reduced pressure. The compound 3a was purified by flash chromatography eluting with ethyl acetate $/ n$-hexane (1:2); yields: $26-73 \%$.

2-Amino-5-trifluoromethyl-1,8-naphthyridine (3a): Brown solid; yield $26 \%$; m.p. $126-128{ }^{\circ} \mathrm{C} .{ }^{1} \mathrm{H}-$ NMR (200 MHz, $\left.\mathrm{CDCl}_{3}\right): \delta=8.91(\mathrm{~d}, J=5 \mathrm{~Hz}, 1 \mathrm{H}, \mathrm{H}-7), 8.08\left(\mathrm{dq}, J_{1}=2, J_{2}=9 \mathrm{~Hz}, 1 \mathrm{H}, \mathrm{H}-4\right), 7.41$ $(\mathrm{d}, J=5 \mathrm{~Hz}, 1 \mathrm{H}, \mathrm{H}-6), 6.94(\mathrm{~d}, J=9 \mathrm{~Hz}, 1 \mathrm{H}, \mathrm{H}-3), 6.43\left(\mathrm{~s}, 2 \mathrm{H}, \mathrm{NH}_{2}\right) .{ }^{13} \mathrm{C}-\mathrm{NMR}\left(100 \mathrm{MHz}, \mathrm{CDCl}_{3}\right)$ : $\delta=159.9$ (C-7), 156.8 (C-8a), $152.0(\mathrm{C}-2), 135.1$ (q, $\left.{ }^{2} J=31 \mathrm{~Hz}, \mathrm{C}-5\right), 134.2$ (C-4), 123.1 (q, $\left.{ }^{1} J=275 \mathrm{~Hz}, \mathrm{CF}_{3}\right), 114.8$ (C-3), 114.5 (q, $\left.{ }^{3} \mathrm{~J}=5 \mathrm{~Hz}, \mathrm{C}-6\right), 112.5$ (C-4a). GC-MS (EI, $\left.70 \mathrm{eV}\right) \mathrm{m} / \mathrm{z}: 214$ $\left(\mathrm{M}^{+}, 100\right), 194$ (52), 69 (10\%). Anal. Calcd. For $\mathrm{C}_{9} \mathrm{H}_{6} \mathrm{~F}_{3} \mathrm{~N}_{3}$ (213.05): C, 50.71; H, 2.84; N, 19.71\%. Found: C, 50.77; H, 2.92; N, 19.81\%.

2-Amino-5-trifluoromethyl-7-methyl-1,8-naphthyridine (3b): Yellow solid; yield 39\%; m.p. 200-202 ${ }^{\circ} \mathrm{C}$ (lit. [22] 195-197 ${ }^{\circ} \mathrm{C}$ ). ${ }^{1} \mathrm{H}-\mathrm{NMR}\left(200 \mathrm{MHz}, \mathrm{DMSO}-d_{6}\right): \delta=8.01$ (dq, $J_{1}=2, J_{2}=9 \mathrm{~Hz}$, $1 \mathrm{H}, \mathrm{H}-4), 7.43$ (s, 1H, H-6), 7.16 (s, 2H, NH ), 6.97 (d, J=9 Hz, 1H, H-3), $2.64\left(\mathrm{~s}, 3 \mathrm{H}, \mathrm{CH}_{3}\right) .{ }^{13} \mathrm{C}-$ NMR (100 MHz, DMSO- $\left.d_{6}\right): \delta=160.8$ (C-7), 160.7 (C-8a), $157.0(\mathrm{C}-2), 132.9$ (q, $\left.{ }^{2} J=31 \mathrm{~Hz}, \mathrm{C}-5\right)$, 132.4 (C-4), 123.5 (q, $\left.{ }^{1} J=275 \mathrm{~Hz}, \mathrm{CF}_{3}\right), 114.1$ (C-3), 113.6 (q, $\left.{ }^{3} J=5 \mathrm{~Hz}, \mathrm{C}-6\right), 108.8$ (C-4a), 24.8 $\left(\mathrm{CH}_{3}\right)$. GC-MS (EI, $\left.70 \mathrm{eV}\right) \mathrm{m} / \mathrm{z} 227\left(\mathrm{M}^{+}, 100\right), 210$ (3), 200 (37), 158 (3), 131 (5\%).

2-Amino-5-trifluoromethyl-7-phenyl-1,8-naphthyridine (3c): Beige solid; yield 69\%; m.p. 255-257 ${ }^{\circ} \mathrm{C}$. ${ }^{1} \mathrm{H}-\mathrm{NMR}\left(200 \mathrm{MHz}, \mathrm{DMSO}-d_{6}\right): \delta=8.28\left(\mathrm{dd}, J_{1}=2, J_{2}=8 \mathrm{~Hz}, 2 \mathrm{H}, \mathrm{Ph}\right), 8.06\left(\mathrm{dq}, J_{1}=2, J_{2}=9 \mathrm{~Hz}\right.$, 1H, H-4), 8.03 (s, 1H, H-6), 7.58-7.53 (m, 3H, Ph), 7.18 (s, 2H, NH 2$), 7.05$ (d, J=9 Hz, 1H, H-3). ${ }^{13} \mathrm{C}-$ NMR (100 MHz, DMSO- $\left.d_{6}\right): \delta=161.0$ (C-7), 157.5 (C-8a), 157.2 (C-2), 137.7 (C-Ph), 134.0 (q, $\left.{ }^{2} J=31 \mathrm{~Hz}, \mathrm{C}-5\right), 132.3$ (q, $\left.{ }^{4} J=2 \mathrm{~Hz}, \mathrm{C}-4\right), 129.8,128.5,127.0$ (5 C-Ph), 123.2 (q, $\left.{ }^{1} J=275 \mathrm{~Hz}, \mathrm{CF}_{3}\right)$, 114.8 (C-3), 110.2 (q, $\left.{ }^{3} J=5 \mathrm{~Hz}, \mathrm{C}-6\right), 110.0$ (q, $\left.{ }^{3} \mathrm{~J}=2 \mathrm{~Hz}, \mathrm{C}-4 \mathrm{a}\right)$. GC-MS (EI, $\left.70 \mathrm{eV}\right) \mathrm{m} / \mathrm{z}: 290\left(\mathrm{M}^{+}\right.$, 100), 270 (23\%). Anal. Calcd. For $\mathrm{C}_{15} \mathrm{H}_{10} \mathrm{~F}_{3} \mathrm{~N}_{3}$ (289.08): C, 62.28; H, 3.48; N, 14.53\%. Found: C, $62.12 ; \mathrm{H}, 3.60 ; \mathrm{N}, 14.30 \%$.

2-Amino-5-trifluoromethyl-7-(4-methylphenyl)-1,8-naphthyridine (3d): Yellow solid; yield 51\%; m.p. 219-221 ${ }^{\circ} \mathrm{C} .{ }^{1} \mathrm{H}-\mathrm{NMR}\left(200 \mathrm{MHz}, \mathrm{DMSO}-d_{6}\right): \delta=8.19$ (d, $\left.J=8 \mathrm{~Hz}, 2 \mathrm{H}, \mathrm{Ph}\right), 8.09$ (dd, $J_{1}=2$, $J=9 \mathrm{~Hz}, 1 \mathrm{H}, \mathrm{H}-4), 7.98$ (s, 1H, H-6), 7.35 (d, $J=8 \mathrm{~Hz}, 2 \mathrm{H}, \mathrm{Ph}), 7.20$ (s, 2H, NH ), 7.08 (d, $J=9 \mathrm{~Hz}$, $1 \mathrm{H}, \mathrm{H}-3), 2.40\left(\mathrm{~s}, 3 \mathrm{H}, \mathrm{CH}_{3}\right) .{ }^{13} \mathrm{C}-\mathrm{NMR}\left(100 \mathrm{MHz}, \mathrm{DMSO}-d_{6}\right): \delta=160.9$ (C-7), 157.4 (C-8a), 157.2 (C-2), 139.5, 134.9 (2 C-Ph), 133.8 (q, $\left.{ }^{2} J=31 \mathrm{~Hz}, \mathrm{C}-5\right), 132.1$ (C-4), 129.1, 126.8 (4 C-Ph), 123.2 (q, $\left.{ }^{1} J=275 \mathrm{~Hz}, \mathrm{CF}_{3}\right), 114.5$ (C-3), 109.8 (q, $\left.{ }^{3} \mathrm{~J}=5 \mathrm{~Hz}, \mathrm{C}-6\right), 99.5$ (C-4a), $20.5\left(\mathrm{CH}_{3}\right)$. GC-MS (EI, $\left.70 \mathrm{eV}\right)$ m/z: $303\left(\mathrm{M}^{+}, 100\right), 276(12), 234$ (14\%). Anal. Calcd. For $\mathrm{C}_{16} \mathrm{H}_{12} \mathrm{~F}_{3} \mathrm{~N}_{3}$ (303.1): C, 63.36; H, 3.99; N, 13.86\%. Found: C, 63.44; H, 4.06; N, 14.01\%.

2-Amino-5-trifluoromethyl-7-(4-methoxyphenyl)-1,8-naphthyridine (3e): Beige solid; yield 53\%; m.p. 210-212 ${ }^{\circ} \mathrm{C} .{ }^{1} \mathrm{H}-\mathrm{NMR}\left(200 \mathrm{MHz}, \mathrm{DMSO}-d_{6}\right): \delta=8.32(\mathrm{~d}, J=8 \mathrm{~Hz}, 2 \mathrm{H}, \mathrm{Ph}), 8.10\left(\mathrm{dq}, J_{1}=2, J_{2}=9 \mathrm{~Hz}\right.$, $1 \mathrm{H}, \mathrm{H}-4), 8.03$ (s, 1H, H-6), 7.44 (s, 2H, NH$)_{2}, 7.13$ (d, J=8 Hz, 2H, Ph), 7.10 (d, J=9 Hz, 1H, H-3), $3.89\left(\mathrm{~s}, 3 \mathrm{H}, \mathrm{CH}_{3}\right) .{ }^{13} \mathrm{C}-\mathrm{NMR}\left(100 \mathrm{MHz}, \mathrm{DMSO}-d_{6}\right): \delta=161.2(\mathrm{C}-7), 160.9(\mathrm{C}-8 \mathrm{a}), 157.4(\mathrm{C}-\mathrm{Ph})$, 157.3 (C-2), 134.0 (q, $\left.{ }^{2} J=31 \mathrm{~Hz}, \mathrm{C}-5\right), 132.5$ (C-4), 130.2, 128.7 (3 C-Ph), 123.4 (q, ${ }^{1} J=275 \mathrm{~Hz}$, $\left.\mathrm{CF}_{3}\right), 114.5$ (C-3), 114.1 (2 C-Ph), 109.8 (q, $\left.{ }^{3} \mathrm{~J}=5 \mathrm{~Hz}, \mathrm{C}-6\right), 109.6$ (C-4a), $55.2\left(\mathrm{OCH}_{3}\right)$. GC-MS (EI, 
$70 \mathrm{eV}) \mathrm{m} / \mathrm{z}: 320\left(\mathrm{M}^{+}, 100\right), 300$ (22\%). Anal. Calcd. For $\mathrm{C}_{16} \mathrm{H}_{12} \mathrm{~F}_{3} \mathrm{~N}_{3} \mathrm{O}$ (319.09): C, 60.19; H, 3.79; N, $13.16 \%$. Found: C, 59.90; H, 3.89; N, 13.07\%.

2-Amino-5-trifluoromethyl-7-(4-fluorophenyl)-1,8-naphthyridine (3f): Yellow solid; yield 73\%; m.p. 260-262 ${ }^{\circ} \mathrm{C} .{ }^{1} \mathrm{H}-\mathrm{NMR}\left(200 \mathrm{MHz}, \mathrm{DMSO}-d_{6}\right): \delta=8.36-8.32(\mathrm{~m}, 2 \mathrm{H}, \mathrm{FPh}), 8.06$ (dq, $J_{1}=2, J_{2}=9 \mathrm{~Hz}$, $1 \mathrm{H}, \mathrm{H}-4), 8.01$ (s, 1H, H-6), 7.38-7.33 (m, 2H, FPh), 7.14 (s, 2H, NH$), 7.02$ (d, J=9 Hz, 1H, H-3). ${ }^{13} \mathrm{C}-\mathrm{NMR}\left(100 \mathrm{MHz}, \mathrm{DMSO}-d_{6}\right): \delta=163.4\left(\mathrm{~d},{ }^{1} \mathrm{~J}=247 \mathrm{~Hz}, \mathrm{C}-\mathrm{FPh}\right), 161.2(\mathrm{C}-7), 157.3$ (C-8a), 156.5 (C-2), 134.2 (d, $\left.{ }^{4} J=3 \mathrm{~Hz}, \mathrm{C}-\mathrm{FPh}\right), 134.1$ (q, $\left.{ }^{2} J=31 \mathrm{~Hz}, \mathrm{C}-5\right), 132.4$ (C-4), 129.5 (d, ${ }^{3} J=9 \mathrm{~Hz}, 2 \mathrm{C}-$ $\mathrm{FPh}), 123.3$ (q, $\left.{ }^{1} J=275 \mathrm{~Hz}, \mathrm{CF}_{3}\right), 115.6\left(\mathrm{~d},{ }^{2} J=21 \mathrm{~Hz}, 2 \mathrm{C}-\mathrm{FPh}\right), 115.1(\mathrm{C}-3), 110.2$ (q, ${ }^{3} \mathrm{~J}=5 \mathrm{~Hz}, \mathrm{C}-$ 6), 110.0 (q, $\left.{ }^{3} J=2 \mathrm{~Hz}, \mathrm{C}-4 \mathrm{a}\right)$. GC-MS (EI, $70 \mathrm{eV}$ ) m/z: $307\left(\mathrm{M}^{+}, 100\right), 238$ (25), 164 (2), 143 (4\%). Anal. Calcd. For $\mathrm{C}_{15} \mathrm{H}_{9} \mathrm{~F}_{4} \mathrm{~N}_{3}$ (307.07): C, 58.64; H, 2.95; N, 13.68\%. Found: C, 58.48; H, 3.11; N, $13.24 \%$.

2-Amino-7-(4-bromophenyl)-5-trifluoromethyl-1,8-naphthyridine (3g): Yellow solid; yield 73\%; m.p. 285-287 ${ }^{\circ} \mathrm{C} .{ }^{1} \mathrm{H}-\mathrm{NMR}\left(200 \mathrm{MHz}, \mathrm{DMSO}-d_{6}\right): \delta=8.23\left(\mathrm{dd}, J_{1}=2, J_{2}=8 \mathrm{~Hz}, 2 \mathrm{H}, \mathrm{Ph}\right), 8.08\left(\mathrm{dq}, J_{1}=2\right.$, $\left.J_{2}=9 \mathrm{~Hz}, 1 \mathrm{H}, \mathrm{H}-4\right), 8.02(\mathrm{~s}, 1 \mathrm{H}, \mathrm{H}-6), 7.72\left(\mathrm{dd}, J_{1}=2, J_{2}=8 \mathrm{~Hz}, 2 \mathrm{H}, \mathrm{Ph}\right), 7.18\left(\mathrm{~s}, 2 \mathrm{H}, \mathrm{NH}_{2}\right), 7.07$ (d, $J=9 \mathrm{~Hz}, 1 \mathrm{H}, \mathrm{H}-3) .{ }^{13} \mathrm{C}-\mathrm{NMR}\left(100 \mathrm{MHz}, \mathrm{DMSO}-d_{6}\right): \delta=161.3$ (C-7), 157.3 (C-8a), 156.4 (C-2), $136.9(\mathrm{C}-\mathrm{Ph}), 134.2$ (q, $\left.{ }^{2} J=31 \mathrm{~Hz}, \mathrm{C}-5\right), 132.5$ (C-4), 131.7, 129.2, 123.9 (5 C-Ph), 123.4 (q, $\left.{ }^{1} J=275 \mathrm{~Hz}, \mathrm{CF}_{3}\right), 115.3$ (C-3), 110.4 (C-4a), 110.2 (q, $\left.{ }^{3} \mathrm{~J}=5 \mathrm{~Hz}, \mathrm{C}-6\right)$. GC-MS (EI, $\left.70 \mathrm{eV}\right) \mathrm{m} / z: 367$ $\left(\mathrm{M}^{+}, 100\right), 298$ (12), 288 (50), 144 (17\%). Anal. Calcd. For $\mathrm{C}_{15} \mathrm{H}_{9} \mathrm{BrF}_{3} \mathrm{~N}_{3}$ (366.99): C, 48.94; H, 2.46; N, 11.41\%. Found: C, 49.04; H, 2.54; N, 11.37\%.

2-Amino-5-trifluoromethyl-7-(4-nitrophenyl)-1,8-naphthyridine (3h): Yellow solid; yield 63\%; m.p. > $340{ }^{\circ} \mathrm{C} .{ }^{1} \mathrm{H}-\mathrm{NMR}\left(200 \mathrm{MHz}, \mathrm{DMSO}-d_{6}\right): \delta=8.53(\mathrm{~d}, J=8 \mathrm{~Hz}, 2 \mathrm{H}, \mathrm{Ph}), 8.35(\mathrm{~d}, J=8 \mathrm{~Hz}, 2 \mathrm{H}, \mathrm{Ph})$, $8.06\left(\mathrm{dd}, J_{1}=2, J=9 \mathrm{~Hz}, 1 \mathrm{H}, \mathrm{H}-4\right), 8.02(\mathrm{~s}, 1 \mathrm{H}, \mathrm{H}-6), 7.20$ (s, 2H, NH 2$), 7.06$ (d, $\left.J=9 \mathrm{~Hz}, 1 \mathrm{H}, \mathrm{H}-3\right)$. ${ }^{13} \mathrm{C}-\mathrm{NMR}\left(100 \mathrm{MHz}, \mathrm{DMSO}-d_{6}\right): \delta=161.1$ (C-7), 157.1 (C-8a), 155.0 (C-2), 148.0, 143.4 (2 C-Ph), 134.3 (q, $\left.{ }^{2} J=31 \mathrm{~Hz}, \mathrm{C}-5\right), 132.1$ (C-4), 128.2, 123.5 (4 C-Ph), 123.0 (q, ${ }^{1} J=275 \mathrm{~Hz}, \mathrm{CF}_{3}$ ), 115.7 (C3), 110.9 (C-4a), 109.8 (q, $\left.{ }^{3} J=2 \mathrm{~Hz}, \mathrm{C}-6\right)$. GC-MS (EI, $\left.70 \mathrm{eV}\right) \mathrm{m} / z: 334\left(\mathrm{M}^{+}, 100\right), 288(97), 144$ (17\%). Anal. Calcd. For $\mathrm{C}_{15} \mathrm{H}_{9} \mathrm{~F}_{3} \mathrm{~N}_{4} \mathrm{O}_{2}$ (334.07): C, 53.90; H, 2.71; N, 16.76\%. Found: C, 53.85; H, $2.78 ; \mathrm{N}, 16.52 \%$.

2-Amino-5-trifluoromethyl-7-(2-furyl)-1,8-naphthyridine (3i): Yellow solid, yield 38\%; m.p. 230-232 ${ }^{\circ} \mathrm{C}$. ${ }^{1} \mathrm{H}-\mathrm{NMR}\left(200 \mathrm{MHz}, \mathrm{DMSO}-d_{6}\right): \delta=7.98\left(\mathrm{~d}, J=4 \mathrm{~Hz}, 1 \mathrm{H}\right.$, furyl), 7.89 (dq, $J_{1}=2, J_{2}=9 \mathrm{~Hz}, 1 \mathrm{H}$, $\mathrm{H}-4), 7.80$ (s, 1H, H-6), 7.40 (d, $J=5 \mathrm{~Hz}, 1 \mathrm{H}$, furyl), 7.17 (s, 2H, NH ), 7.04 (d, $J=9 \mathrm{~Hz}, 1 \mathrm{H}, \mathrm{H}-3)$, 6.75 (t, $J=4 \mathrm{~Hz}, 1 \mathrm{H}$, furyl). ${ }^{13} \mathrm{C}-\mathrm{NMR}\left(100 \mathrm{MHz}, \mathrm{DMSO}-d_{6}\right): \delta=160.9$ (C-7), 157.0 (C-8a), 152.3 (C-2), 149.5, 145.1, (2 C-furyl), 133.9 (q, $\left.{ }^{2} J=31 \mathrm{~Hz}, \mathrm{C}-5\right), 132.3$ (q, $\left.{ }^{4} J=2 \mathrm{~Hz}, \mathrm{C}-4\right), 123.0$ (q, ${ }^{1} J=$ $275 \mathrm{~Hz}, \mathrm{CF}_{3}$ ), 114.5 (C-3), 112.4, 111.1 (2 C-furyl), 109.8 (q, ${ }^{3} \mathrm{~J}=2 \mathrm{~Hz}, \mathrm{C}-4 \mathrm{a}$ ), 108.6 (q, ${ }^{3} \mathrm{~J}=5 \mathrm{~Hz}, \mathrm{C}-$ 6). GC-MS (EI, $70 \mathrm{eV}) \mathrm{m} / \mathrm{z}: 279\left(\mathrm{M}^{+}, 100\right), 251$ (25), 223 (12\%). Anal. Calcd. For $\mathrm{C}_{13} \mathrm{H}_{8} \mathrm{~F}_{3} \mathrm{~N}_{3} \mathrm{O}$ (279.06): C, 55.92; H, 2.89; N, 15.05\%. Found: C, 55.48; H, 2.88; N, 14.72\%.

2-Amino-5-trifluoromethyl-7-(2-thienyl)-1,8-naphthyridine (3j): Yellow solid; yield 60\%; m.p. 260$262{ }^{\circ} \mathrm{C} .{ }^{1} \mathrm{H}-\mathrm{NMR}\left(200 \mathrm{MHz}, \mathrm{DMSO}-d_{6}\right): \delta=8.12\left(\mathrm{~d}, J=4 \mathrm{~Hz}, 1 \mathrm{H}\right.$, thienyl), $8.07\left(\mathrm{dq}, J_{1}=2, J_{2}=9 \mathrm{~Hz}\right.$, $1 \mathrm{H}, \mathrm{H}-4), 8.04$ (s, 1H, H-6), 7.78 (d, $J=5 \mathrm{~Hz}, 1 \mathrm{H}$, thienyl), 7.26 (t, $J=4 \mathrm{~Hz}, 1 \mathrm{H}$, thienyl), 7.23 (s, 2H, 
$\mathrm{NH}_{2}$ ), 7.07 (d, $\left.J=9 \mathrm{~Hz}, 1 \mathrm{H}, \mathrm{H}-3\right) .{ }^{13} \mathrm{C}-\mathrm{NMR}\left(100 \mathrm{MHz}, \mathrm{DMSO}-d_{6}\right): \delta=161.1$ (C-7), 157.0 (C-8a), 153.2 (C-2), 143.9 (C-thienyl), 133.9 (q, $\left.{ }^{2} J=31 \mathrm{~Hz}, \mathrm{C}-5\right), 132.3$ (q, $\left.{ }^{4} J=2 \mathrm{~Hz}, \mathrm{C}-4\right), 130.0,128.3$, 127.5 (3 C-thienyl), 123.1 (q, ${ }^{1} J=275 \mathrm{~Hz}, \mathrm{CF}_{3}$ ), 114.3 (C-3), 109.9 (q, $\left.{ }^{3} J=2 \mathrm{~Hz}, \mathrm{C}-4 \mathrm{a}\right), 109.3$ (q, ${ }^{3} J=$ $5 \mathrm{~Hz}, \mathrm{C}-6)$. GC-MS (EI, $70 \mathrm{eV}$ ) m/z: 295 (M+100), 268 (25), 226 (4\%). Anal. Calcd. For $\mathrm{C}_{13} \mathrm{H}_{8} \mathrm{~F}_{3} \mathrm{~N}_{3} \mathrm{~S}$ (295.04): C, 52.88; H, 2.73; N, 14.23\%. Found: C, 52.94; H, 2.85; N, 14.10\%.

3.5. General procedure for the synthesis of 2-amino-5-trifluoromethyl-7,8-dihydro-6Hcyclopenta[b][1,8]-naphthyridine (3k)

To a stirred mixture of $\mathrm{H}_{3} \mathrm{PO}_{4}(0.8 \mathrm{~mL})$ and $\mathrm{P}_{2} \mathrm{O}_{5}(1.2 \mathrm{~g})(\mathrm{PPA})$ at $90{ }^{\circ} \mathrm{C}, \mathbf{2 k}(0.27 \mathrm{~g}, 1 \mathrm{mmol})$ was added. The reaction mixture was stirred for an additional $20 \mathrm{~h}$. After cooling, the reaction mixture was treated with crushed ice and with concentrated $\mathrm{NH}_{4} \mathrm{OH}$ until the $\mathrm{pH}$ was 8 . The compound $\mathbf{3 k}$ was isolated of the solution by filtration at reduced pressure as a brown solid, in 78\% yield, m.p. 172-174 ${ }^{\circ} \mathrm{C}$. ${ }^{1} \mathrm{H}-\mathrm{NMR}\left(200 \mathrm{MHz}, \mathrm{DMSO}-d_{6}\right): \delta=8.00\left(\mathrm{dq}, J_{1}=2, J_{2}=9 \mathrm{~Hz}, 1 \mathrm{H}, \mathrm{H}-4\right), 6.91(\mathrm{~d}, J=9 \mathrm{~Hz}, 1 \mathrm{H}$, $\mathrm{H}-3), 6.80$ (s, 2H, $\mathrm{NH}_{2}$ ), 3.18-3.14 (m, 2H, $\left.\mathrm{CH}_{2}\right), 3.02$ (t, $\left.J=9 \mathrm{~Hz}, 2 \mathrm{H}, \mathrm{CH}_{2}\right), 2.15-2.07\left(\mathrm{~m}, 2 \mathrm{H}, \mathrm{CH}_{2}\right)$. ${ }^{13} \mathrm{C}-\mathrm{NMR}\left(100 \mathrm{MHz}, \mathrm{DMSO}-d_{6}\right): \delta=170.0$ (C-9a), $159.8(\mathrm{C}-2), 156.5$ (C-10a), 132.5 (q, ${ }^{4} \mathrm{~J}=3 \mathrm{~Hz}, \mathrm{C}-$ 4), $128.0\left(\mathrm{q},{ }^{3} J=3 \mathrm{~Hz}, \mathrm{C}-5 \mathrm{a}\right), 127.6\left(\mathrm{q},{ }^{2} \mathrm{~J}=30 \mathrm{~Hz}, \mathrm{C}-5\right), 124.2\left(\mathrm{q},{ }^{1} J=276 \mathrm{~Hz}, \mathrm{CF}_{3}\right), 113.0(\mathrm{C}-3)$, 109.2 (q, $\left.{ }^{3} J=2 \mathrm{~Hz}, \mathrm{C}-4 \mathrm{a}\right), 33.7,29.7$ (q, $\left.{ }^{4} \mathrm{~J}=2 \mathrm{~Hz}\right), 21.9\left(3 \mathrm{CH}_{2}\right)$. GC-MS (EI, $\left.70 \mathrm{eV}\right) \mathrm{m} / \mathrm{z}: 253\left(\mathrm{M}^{+}\right.$, 100), 237 (5), 184 (20\%). Anal. Calcd. For $\mathrm{C}_{12} \mathrm{H}_{10} \mathrm{~F}_{3} \mathrm{~N}_{3}$ (253.08): C, 56.92; H, 3.98; N, 16.59\%. Found: C, 56.80; H, 3.84; N, 16.73\%.

3.6. General procedure for the synthesis of 2-amino-5-trifluoromethyl-cycloalka[b][1,8]naphthyridines $\mathbf{3 1}, \mathbf{3 m}$

To a magnetically stirred solution of 2,6-diaminopyridine $(0.22 \mathrm{~g}, 2 \mathrm{mmol})$ in methanol $(20 \mathrm{~mL})$, a solution of $\mathbf{1} \mathbf{k}-\mathbf{l}(2 \mathrm{mmol})$ in methanol $(20 \mathrm{~mL})$ was added drop wise at $0{ }^{\circ} \mathrm{C}$ over a period of $2 \mathrm{~h}$. The mixture was refluxed for an additional $24 \mathrm{~h}$. After the end of the reaction, the solvent was evaporated under reduced pressure. The compounds 3k-l were purified by flash chromatography eluting with ethyl acetate/ $n$-hexane (1:2); yields: $30-33 \%$.

2-Amino-5-trifluoromethyl-6,7,8,9-tetrahydrobenzo[b][1,8]naphthyridine (31): Brown solid, yield $33 \%$; m.p. $128-130{ }^{\circ} \mathrm{C} .{ }^{1} \mathrm{H}-\mathrm{NMR}\left(200 \mathrm{MHz}, \mathrm{DMSO}-d_{6}\right): \delta=8.07$ (dq, $\left.J_{1}=2, J_{2}=9 \mathrm{~Hz}, 1 \mathrm{H}, \mathrm{H}-4\right)$, $6.93(\mathrm{~d}, J=9 \mathrm{~Hz}, 1 \mathrm{H}, \mathrm{H}-3), 6.85\left(\mathrm{~s}, 2 \mathrm{H}, \mathrm{NH}_{2}\right), 2.99-2.96\left(\mathrm{~m}, 4 \mathrm{H}, \mathrm{CH}_{2}\right), 1.90-1.78\left(\mathrm{~m}, 4 \mathrm{H}, \mathrm{CH}_{2}\right) .{ }^{13} \mathrm{C}-$ NMR (100 MHz, DMSO- $d_{6}$ ): $\delta=160.9$ (C-2), 159.8 (C-10a), 154.6 (C-9a), 132.7 (q, $\left.{ }^{4} \mathrm{~J}=3 \mathrm{~Hz}, \mathrm{C}-4\right)$, 130.3 (q, ${ }^{2} J=28 \mathrm{~Hz}, \mathrm{C}-5$ ), 124.7 (q, ${ }^{1} J=278 \mathrm{~Hz}, \mathrm{CF}_{3}$ ), 124.5 (C-5a), 114.0 (C-3), 110.5 (q, ${ }^{3} \mathrm{~J}=2 \mathrm{~Hz}$, C-4a), 33.8, 25.6, 22.0, $21.2\left(4 \mathrm{CH}_{2}\right)$. GC-MS (EI, $\left.70 \mathrm{eV}\right) \mathrm{m} / \mathrm{z}: 268\left(\mathrm{M}^{+}, 100\right), 248$ (20), 198 (9\%). Anal. Calcd. For $\mathrm{C}_{13} \mathrm{H}_{12} \mathrm{~F}_{3} \mathrm{~N}_{3}$ (267.1): C, 58.42; H, 4.53; N, 15.72\%. Found: C, 58.59; H, 4.65; N, $15.61 \%$.

2-Amino-5-trifluoromethyl-7,8,9,10-tetrahydro-6H-cyclohepta[b][1,8]naphthyridine $\quad(\mathbf{3 m})$ : Brown solid; yield 30\%; m.p. 93-95 ${ }^{\circ} \mathrm{C} .{ }^{1} \mathrm{H}-\mathrm{NMR}\left(200 \mathrm{MHz}, \mathrm{CDCl}_{3}\right): \delta=8.20$ (d, $\left.J=9 \mathrm{~Hz}, 1 \mathrm{H}, \mathrm{H}-4\right), 7.75$ $(\mathrm{d}, J=9 \mathrm{~Hz}, 1 \mathrm{H}, \mathrm{H}-3), 7.19$ (s, 2H, NH$), 3.21-3.00\left(\mathrm{~m}, 4 \mathrm{H}, \mathrm{CH}_{2}\right), 1.79-1.68\left(\mathrm{~m}, 6 \mathrm{H}, \mathrm{CH}_{2}\right) .{ }^{13} \mathrm{C}-\mathrm{NMR}$ $\left(100 \mathrm{MHz}, \mathrm{CDCl}_{3}\right): \delta=158.8(\mathrm{C}-2), 156.5$ (C-11a), 154.5 (C-10a), 140.7 (C-5a), 134.6 (C-4), 131.2 
(q, $\left.{ }^{2} J=30 \mathrm{~Hz}, \mathrm{C}-5\right), 123.9\left(\mathrm{q},{ }^{1} J=278 \mathrm{~Hz}, \mathrm{CF}_{3}\right), 113.5$ (C-3), 97.3 (C-4a), 39.0, 38.9, 29.3, 27.5, 26.2 $\left(5 \mathrm{CH}_{2}\right)$. GC-MS (EI, $\left.70 \mathrm{eV}\right) \mathrm{m} / z: 281\left(\mathrm{M}^{+}, 100\right), 265$ (15), 252 (30), 212 (4\%). Anal. Calcd. For $\mathrm{C}_{14} \mathrm{H}_{14} \mathrm{~F}_{3} \mathrm{~N}_{3}$ (281.1): C, 59.78; H, 5.02; N, 14.94\%. Found: C, 59.69; H, 4.88; N, 15.01\%.

3.7. General procedure for the synthesis of 2-acetylamino-7-(aryl/heteroaryl)-5-trifluoromethyl-1,8naphthyridines $\mathbf{4}$

A suspension of $2 \mathrm{mmol}$ of amino derivatives $\mathbf{3 c}, \mathbf{3 f}, \mathbf{3 g}, \mathbf{3 i}$ or $\mathbf{3 j}$ in $5 \mathrm{~mL}$ of acetic anhydride was refluxed at $160{ }^{\circ} \mathrm{C}$ for $2 \mathrm{~h}$. After cooling, the solids were collected and washed with water to give their acetamide derivatives 4 , in $82-93 \%$ yields.

2-Acetylamino-5-trifluoromethyl-7-phenyl-1,8-naphthyridine (4c): Beige solid; yield 74\%; m.p. 224$226{ }^{\circ} \mathrm{C} .{ }^{1} \mathrm{H}-\mathrm{NMR}\left(200 \mathrm{MHz}, \mathrm{DMSO}-d_{6}\right): \delta=11.34$ (s, $\left.1 \mathrm{H}, \mathrm{NH}\right), 8.54-850$ (m, 2H, H-3, H-4), 8.40 (s, $1 \mathrm{H}, \mathrm{H}-6), 8.37-835$ (m, 2H, Ph), 760-7.58 (m, 3H, Ph), $2.22\left(\mathrm{CH}_{3}\right) .{ }^{13} \mathrm{C}-\mathrm{NMR}\left(100 \mathrm{MHz}, \mathrm{DMSO}-d_{6}\right)$ : $\delta=171.6(\mathrm{C}=0), 170.0$ (C-8a), 158.7 (C-7), 155.5 (C-2), 136.9 (C-Ph), 135.4 (q, $\left.{ }^{2} J=33 \mathrm{~Hz}, \mathrm{C}-5\right)$, 134.4 (C-4), 130.4, 128.7, 127.3 (5 C-Ph), 122.9 (q, $\left.{ }^{1} J=275 \mathrm{~Hz}, \mathrm{CF}_{3}\right), 115.9(\mathrm{C}-3), 114.4$ (q, ${ }^{3} \mathrm{~J}=5 \mathrm{~Hz}$, C-6), 113.1 (C-4a), 23.9 ( $\mathrm{CH}_{3}$ ). GC-MS (EI, $\left.70 \mathrm{eV}\right) \mathrm{m} / z$ : 331 (40), 288 (M+, 100), 262 (15), 219 (9\%). Anal. Calcd. For $\mathrm{C}_{17} \mathrm{H}_{12} \mathrm{~F}_{3} \mathrm{~N}_{3} \mathrm{O}(331.09) \mathrm{C}, 61.63 ; \mathrm{H}, 3.65 ; \mathrm{N}, 12.68 \%$. Found: $\mathrm{C}, 61.49 ; \mathrm{H}, 3.50$; N, $12.78 \%$.

2-Acetylamino-5-trifluoromethyl-7-(4-fluorophenyl)-1,8-naphthyridine (4f): Beige solid; yield 83\%; m.p. $263-265{ }^{\circ} \mathrm{C} .{ }^{1} \mathrm{H}-\mathrm{NMR}\left(200 \mathrm{MHz}, \mathrm{CDCl}_{3}\right): \delta=11.21$ (s, $\left.1 \mathrm{H}, \mathrm{NH}\right), 8.50$ (d, J=9 Hz, 1H, H-3), 8.45 (d, $J=9 \mathrm{~Hz}, 1 \mathrm{H}, \mathrm{H}-4), 8.40-8.37$ (m, 2H, FPh), 8.33 (s, 1H, H-6), 7.39-7.35 (m, 2H, FPh), 2.23 (s, 3H, $\left.\mathrm{CH}_{3}\right) .{ }^{13} \mathrm{C}-\mathrm{NMR}\left(100 \mathrm{MHz}, \mathrm{DMSO}-d_{6}\right): \delta=170.0(\mathrm{C}=0), 163.6\left(\mathrm{~d},{ }^{1} J=248 \mathrm{~Hz}, \mathrm{C}-\mathrm{FPh}\right), 157.6$ (C-8a), 155.0 (C-7), 154.9 (C-2), 135.5 (q, $\left.{ }^{2} J=33 \mathrm{~Hz}, \mathrm{C}-5\right), 134.3$ (C-4), 133.4 (d, $\left.{ }^{4} J=3 \mathrm{~Hz}, \mathrm{C}-\mathrm{FPh}\right)$, $129.6\left(\mathrm{~d},{ }^{3} J=9 \mathrm{~Hz}, 2 \mathrm{C}-\mathrm{FPh}\right), 122.7$ (q, $\left.{ }^{1} \mathrm{~J}=275 \mathrm{~Hz}, \mathrm{CF}_{3}\right), 115.8(\mathrm{C}-6), 115.6$ (d, ${ }^{2} J=21 \mathrm{~Hz}, 2 \mathrm{C}-$ FPh), 114.2 (C-3), 113.0 (C-4a), $23.8\left(\mathrm{CH}_{3}\right)$. GC-MS (EI, $\left.70 \mathrm{eV}\right)$ m/z: 349 (45), $307\left(\mathrm{M}^{+}, 100\right), 280$ (25), 238 (15\%). Anal. Calcd. For $\mathrm{C}_{17} \mathrm{H}_{11} \mathrm{~F}_{4} \mathrm{~N}_{3} \mathrm{O}$ (349.08): C, 58.46; H, 3.17; N, 12.03\%. Found: C, $58.28 ; \mathrm{H}, 3.02 ; \mathrm{N}, 12.15 \%$.

2-Acetylamino-7-(4-bromophenyl)-5-trifluoromethyl-1,8-naphthyridine (4g): Beige solid; yield 93\%; m.p. $222-224^{\circ} \mathrm{C} .{ }^{1} \mathrm{H}-\mathrm{NMR}\left(200 \mathrm{MHz}, \mathrm{CDCl}_{3}+\mathrm{TFA}\right): \delta=11.34$ (s, $\left.1 \mathrm{H}, \mathrm{NH}\right), 8.90$ (d, $J=9 \mathrm{~Hz}, 1 \mathrm{H}, \mathrm{H}-$ 3), 8.45 (s, 1H, H-6), 8.18 (d, $J=9 \mathrm{~Hz}, 2 \mathrm{H}, \mathrm{Ph}), 7.80$ (d, $J=9 \mathrm{~Hz}, 2 \mathrm{H}, \mathrm{Ph}), 7.74$ (d, J=9 Hz, 1H, H4), $2.54\left(\mathrm{~s}, 3 \mathrm{H}, \mathrm{CH}_{3}\right) .{ }^{13} \mathrm{C}-\mathrm{NMR}\left(100 \mathrm{MHz}, \mathrm{CDCl}_{3}+\mathrm{TFA}\right): \delta=176.0(\mathrm{C}=0), 169.8(\mathrm{C}-8 \mathrm{a}), 152.1(\mathrm{C}-$ 7), 145.4 (C-2), 143.0 (C-Ph), 137.7 (q, $\left.{ }^{2} J=33 \mathrm{~Hz}, \mathrm{C}-5\right), 133.5$ (C-4), 133.0, 129.7, 128.8, (5 C-Ph), $121.6\left(\mathrm{q},{ }^{1} J=275 \mathrm{~Hz}, \mathrm{CF}_{3}\right), 118.2\left(\mathrm{q},{ }^{3} J=5 \mathrm{~Hz}, \mathrm{C}-6\right), 114.1(\mathrm{C}-3), 113.3(\mathrm{C}-4 \mathrm{a}), 24.2\left(\mathrm{CH}_{3}\right)$. GC-MS (EI, $70 \mathrm{eV}) \mathrm{m} / z$ : 409 (42), $367\left(\mathrm{M}^{+}, 100\right), 339$ (5), 296 (6), 288 (42\%). Anal. Calcd. For $\mathrm{C}_{17} \mathrm{H}_{11} \mathrm{BrF}_{3} \mathrm{~N}_{3} \mathrm{O}$ (409.1): C, 49.78; H, 2.70; N, 10.24\%. Found: C, 49.61; H, 2.58; N, 10.33\%.

2-Acetylamino-5-trifluoromethyl-7-(2-furyl)-1,8-naphthyridine (4i): Yellow solid; yield 82\%; m.p. 221-223 ${ }^{\circ} \mathrm{C} .{ }^{1} \mathrm{H}-\mathrm{NMR}\left(200 \mathrm{MHz}, \mathrm{CDCl}_{3}\right): \delta=9.74$ (s, $\left.1 \mathrm{H}, \mathrm{NH}\right), 8.60$ (d, $\left.J=9 \mathrm{~Hz}, 1 \mathrm{H}, \mathrm{H}-3\right), 8.41$ (d, $J=9 \mathrm{~Hz}, 1 \mathrm{H}, \mathrm{H}-4), 8.10$ (s, 1H, H-6), 7.65 (s, 1H, furyl), 7.45 (d, $J=3 \mathrm{~Hz}, 1 \mathrm{H}$, furyl), $6.62(\mathrm{t}$, $J=2 \mathrm{~Hz}, 1 \mathrm{H}$, furyl), 2.29 (s, 3H, $\left.\mathrm{CH}_{3}\right) .{ }^{13} \mathrm{C}-\mathrm{NMR}\left(100 \mathrm{MHz}, \mathrm{CDCl}_{3}\right): \delta=176.2(\mathrm{C}=0), 169.8(\mathrm{C}-8 \mathrm{a})$, 
152.5 (C-7), 151.8 (C-2), 145.3 (2 C-furyl), 136.0 (q, ${ }^{2} J=32 \mathrm{~Hz}, \mathrm{C}-5$ ), 135.9 (C-4), 122.8 (q, $\left.{ }^{1} J=275 \mathrm{~Hz}, \mathrm{CF}_{3}\right), 115.8$ (C-3), 114.3 (C-4a), 113.8 (q, $\left.{ }^{3} \mathrm{~J}=5 \mathrm{~Hz}, \mathrm{C}-6\right), 112.9,112.7$ (2 C-furyl), 24.5 $\left(\mathrm{CH}_{3}\right)$. GC-MS (EI, $\left.70 \mathrm{eV}\right) \mathrm{m} / z: 321(50), 306(5), 279(\mathrm{M}+, 100), 251$ (10\%). Anal. Calcd. For $\mathrm{C}_{15} \mathrm{H}_{10} \mathrm{~F}_{3} \mathrm{~N}_{3} \mathrm{O}_{2}$ (321.07) C, 56.08; H, 3.14; N, 13.08\%. Found: C, 56.00; H, 3.03; N, 13.18\%.

2-Acetylamino-5-trifluoromethyl-7-(2-thienyl)-1,8-naphthyridine (4j): Beige solid; yield 90\%; m.p. 225-227 ${ }^{\circ} \mathrm{C} .{ }^{1} \mathrm{H}-\mathrm{NMR}\left(200 \mathrm{MHz}, \mathrm{CDCl}_{3}\right): \delta=9.76(\mathrm{~s}, 1 \mathrm{H}, \mathrm{NH}), 8.59(\mathrm{~d}, J=9 \mathrm{~Hz}, 1 \mathrm{H}, \mathrm{H}-3), 8.40(\mathrm{~d}$, $J=9 \mathrm{~Hz}, 1 \mathrm{H}, \mathrm{H}-4), 7.98$ (s, 1H, H-6), 7.84 (s, 1H, thienyl), 7.54 (d, $J=4 \mathrm{~Hz}, 1 \mathrm{H}$, thienyl), 7.16 (d, $J=3 \mathrm{~Hz}, 1 \mathrm{H}$, thienyl), $2.29\left(\mathrm{~s}, 3 \mathrm{H}, \mathrm{CH}_{3}\right) \cdot{ }^{13} \mathrm{C}-\mathrm{NMR}\left(100 \mathrm{MHz}, \mathrm{CDCl}_{3}\right): \delta=176.1(\mathrm{C}=0), 169.8(\mathrm{C}-$ 8a), 155.3 (C-7), 154.9 (C-2), 143.4 (C-thienyl), 135.9 (q, ${ }^{2} J=33$ Hz, C-5), 135.7 (C-4), 130.9, 128.4, 127.9 (3 C-thienyl), 122.9 (q, ${ }^{1} J=275 \mathrm{~Hz}, \mathrm{CF}_{3}$ ), 115.8 (C-3), 114.3 (C-4a), 114.1 (q, ${ }^{3} \mathrm{~J}=5 \mathrm{~Hz}, \mathrm{C}-6$ ), $24.7\left(\mathrm{CH}_{3}\right)$. GC-MS (EI, $\left.70 \mathrm{eV}\right) \mathrm{m} / z$ : 337 (43), $322(5), 295(\mathrm{M}+, 100), 268$ (25\%). Anal. Calcd. For $\mathrm{C}_{15} \mathrm{H}_{10} \mathrm{~F}_{3} \mathrm{~N}_{3} \mathrm{OS}$ (337.05): C, 53.41; H, 2.99\%; N, 12.46. Found: C, 53.33; H, 2.81; N, 12.54\%.

3.8. General procedure for the synthesis of 2-amino-5-trifluoromethyl-7-methyl-1,8-naphthyridine (3b) and 2-amino-7-trifluoromethyl-5-methyl-1,8-naphthyridine (5b)

These compounds were obtained as a yellow solid, a 1:1 mixture of $\mathbf{3 b} \mathbf{b} \mathbf{5 b}$ in $87 \%$ yield, m.p. 179$181{ }^{\circ} \mathrm{C}$. To a stirred mixture of $\mathrm{H}_{3} \mathrm{PO}_{4}(0.8 \mathrm{~mL})$ and $\mathrm{P}_{2} \mathrm{O}_{5}(1.2 \mathrm{~g})(\mathrm{PPA})$ at $90{ }^{\circ} \mathrm{C}, 2 \mathbf{b}(0.24 \mathrm{~g}, 1 \mathrm{mmol})$ was added. The reaction mixture was stirred for an additional $20 \mathrm{~h}$. After cooling, the reaction mixture was treated with crushed ice and with concentrated $\mathrm{NH}_{4} \mathrm{OH}$ until the $\mathrm{pH}$ was 8 . The solution was then extracted with ethyl acetate $(3 \times 20 \mathrm{~mL})$, the combined extracts were dried $\left(\mathrm{MgSO}_{4}\right)$ and evaporated to dryness under a vacuum to obtain the solid mixture of isomers $\mathbf{3 b}: \mathbf{5 b}$.

2-Amino-5-trifluoromethyl-7-methyl-1,8-naphthyridine (3b): Yield 44\%. ${ }^{1} \mathrm{H}-\mathrm{NMR}\left(200 \mathrm{MHz}, \mathrm{CDCl}_{3}\right)$ : $\delta=8.08$ (d, $J=9 \mathrm{~Hz}, 1 \mathrm{H}, \mathrm{H}-4), 7.36$ (s, 1H, H-6), 6.98 (d, $J=9 \mathrm{~Hz}, 1 \mathrm{H}, \mathrm{H}-3), 6.19$ (s, 2H, NH$)_{2}, 2.74$ $\left(\mathrm{s}, 3 \mathrm{H}, \mathrm{CH}_{3}\right) .{ }^{13} \mathrm{C}-\mathrm{NMR}\left(100 \mathrm{MHz}, \mathrm{DMSO}-d_{6}\right): \delta=160.5(\mathrm{C}-7), 159.8(\mathrm{C}-8 \mathrm{a}), 156.5(\mathrm{C}-2), 133.8(\mathrm{q}$, $\left.{ }^{2} J=31 \mathrm{~Hz}, \mathrm{C}-5\right), 133.0(\mathrm{C}-4), 122.6\left(\mathrm{q},{ }^{1} J=275 \mathrm{~Hz}, \mathrm{CF}_{3}\right), 114.2(\mathrm{C}-3), 113.8$ (q, $\left.{ }^{3} \mathrm{~J}=5 \mathrm{~Hz}, \mathrm{C}-6\right)$, 112.9 (C-4a), $24.6\left(\mathrm{CH}_{3}\right)$. GC-MS (EI, $\left.70 \mathrm{eV}\right) \mathrm{m} / z 227$ (M+1 100), 210 (3), 200 (37), 158 (3), 131 (5\%).

2-amino-7-trifluoromethyl-5-methyl-1,8-naphthyridine (5b): Yield 43\%. ${ }^{1} \mathrm{H}-\mathrm{NMR}\left(200 \mathrm{MHz}, \mathrm{CDCl}_{3}\right)$ : $\delta=8.08(\mathrm{~d}, J=9 \mathrm{~Hz}, 1 \mathrm{H}, \mathrm{H}-4), 7.33(\mathrm{~s}, 1 \mathrm{H}, \mathrm{H}-6), 6.90(\mathrm{~d}, J=9 \mathrm{~Hz}, 1 \mathrm{H}, \mathrm{H}-3), 5.98\left(\mathrm{~s}, 2 \mathrm{H}, \mathrm{NH}_{2}\right), 2.67$ $\left(\mathrm{s}, 3 \mathrm{H}, \mathrm{CH}_{3}\right) .{ }^{13} \mathrm{C}-\mathrm{NMR}\left(100 \mathrm{MHz}, \mathrm{DMSO}-d_{6}\right): \delta=160.3(\mathrm{C}-7), 159.8(\mathrm{C}-8 \mathrm{a}), 155.2(\mathrm{C}-2), 148.1\left(\mathrm{q},{ }^{2} \mathrm{~J}\right.$ $=34 \mathrm{~Hz}, \mathrm{C}-7), 146.9$ (C-5), 132.7 (C-4), 120.8 (q, $\left.{ }^{1} J=275 \mathrm{~Hz}, \mathrm{CF}_{3}\right), 114.2$ (C-3), 113.8 (q, ${ }^{3} \mathrm{~J}=5 \mathrm{~Hz}$, C-6), 112.9 (C-4a), $17.5\left(\mathrm{CH}_{3}\right)$. GC-MS (EI, $\left.70 \mathrm{eV}\right)$ m/z: $227\left(\mathrm{M}^{+}, 100\right), 210$ (3), 200 (37), 158 (3), $131(5 \%)$.

\section{Conclusions}

In summary, we have developed a new, simple and convenient route for the preparation of a new series of 7-alkyl(aryl/heteroaryl)-2-amino-5-trifluoromethyl-1,8-naphthyridines 3a-j in moderate to good yields, derived from direct cyclocondensation reactions employing acyclic $\beta$-alkoxyvinyl trifluoromethyl ketones 1a-j and 2,6-DAP, under mild conditions by a conventional one-pot procedure. 
We also developed the synthesis of new 2-amino-5-trifluoromethyl-cycloalka[b][1,8]naphthyridines 31-m as fused heteropolycycles from direct or indirect cyclocondensation reactions of 2-trifluoroacetyl1-methoxycycloalkenes 11-m and 2,6-DAP. Furthermore, we have been able to use acyclic and cyclic ketones $\mathbf{1}$, for the first time, in the synthesis of trifluoromethylated 2-amino-1,8-naphthyridines, which possess a free amino group for further important derivatizations.

\section{Acknowledgements}

The authors thank the Conselho Nacional de Desenvolvimento Científico, CNPq, for the financial support (Proc. Nr. 303.296/2008-9). Fellowships from CAPES and CNPq are also acknowledged.

\section{References and Notes}

1. Goswami, S.; Mukherjee, R.; Mukherjee, R.; Jana, S.; Maity, A.C.; Adak, A.K. Simple and efficient synthesis of 2,7-difunctionalized-1,8-naphthyridines. Molecules 2005, 10, 929-936.

2. Mekheimer, R.A.; Hameed, A.M.A.; Sadek, K.U. 1,8-Naphthyridines II: Synthesis of novel polyfunctionally substituted 1,8-naphthyridinones and their degradation to 6-aminopyridones. ARKIVOC 2007, xiii, 269-281.

3. He, C.; Lippard, S.J. Design and synthesis of multidentate dinucleating ligands based on 1,8naphthyridine. Tetrahedron 2000, 56, 8245-8252.

4. Bouzard, D.; DiCesare, P.; Essiz, M.; Jacquet, J.P.; Ledoussal, B.; Remuzon, P.; Kessler, R.E.; Fung-Tomc, J. Fluoronaphthyridines as antibacterial agents. 4. Synthesis and structure-activity relationships of 5-substituted-6-fluoro-7-(cycloalkylamino)-1,4-dihydro-4-oxo-1,8-naphthyridine3-carboxylic acids. J. Med. Chem. 1992, 35, 518-525.

5. Ferrarini, P.L.; Manera, C.; Mori, C.; Badawneh, M.; Saccomanni, G. Synthesis and evaluation of antimycobacterial activity of 4-phenyl-1,8-naphthyridine derivatives. Farmaco 1998, 53, 741-746.

6. Tsuzuki, Y.; Tomita, K.; Sato, Y.; Kashimoto, S.; Chiba, K. Synthesis and structure-activity relationships of 3-substituted 1,4-dihydro-4-oxo-1-(2-thiazolyl)-1,8-naphthyridines as novel antitumor agents. Bioorg. Med. Chem. Lett. 2004, 14, 3189-3193.

7. Dianzani, C.; Collino, M.; Gallicchio, M.; Di Braccio, M.; Roma, G.; Fantozzi, R. Effects of antiinflammatory $[1,2,4]$ triazolo[4,3-a][1,8]naphthyridine derivatives on human stimulated PMN and endothelial cells: An in vitro study. J. Inflamm. 2006, 3, 4.

8. Roma, G.; Di Braccio, M.; Grossi, G.; Piras, D.; Ballabeni, V.; Tognolini, M.; Bertoni, S.; Barocelli, E. 1,8-Naphthyridines VIII. Novel 5-aminoimidazo[1,2-a][1,8]naphthyridine-6carboxamide and 5-amino[1,2,4]triazolo[4,3-a][1,8] naphthyridine-6-carboxamide derivatives showing potent analgesic or anti-inflammatory activity, respectively, and completely devoid of acute gastrolesivity. Eur. J. Med. Chem. 2010, 45, 352-366.

9. Ferrarini, P.L.; Badawneh, M.; Franconi, F.; Manera, C.; Miceli, M.; Mori, C.; Saccomanni, G. Synthesis and antiplatelet activity of some 2,7-di( $N$-cycloamino)-3-phenyl-1,8-naphthyridine derivatives. Farmaco 2001, 56, 311-318.

10. Santilli, A.A.; Scotese, A.C.; Bauer, R.F.; Bell, S.C. 2-Oxo-1,8-naphthyridine-3-carboxylic acid derivatives with potent gastric antisecretory properties. $J$. Med. Chem. 1987, 30, 2270-2277. 
11. Ferrarini, P.L.; Mori, C.; Tellini, N. Synthesis and local anesthetic activity of (E)- and (Z)diethylaminoethyliminothers of 1,8-naphtyridine. Farmaco 1990, 45, 385-389.

12. Leonard, J.T.; Gangadhar, R.; Gnanasam, S.K.; Ramachandran, S.; Saravanan, M.; Sridhar, S.K. Synthesis and pharmacological activities of 1,8-naphthyridine derivatives. Biol. Pharm. Bull. 2002, 25, 798-802.

13. Ferrarini, P.L.; Mori, C.; Calderone, V.; Calzolari, L.; Nieri, P.; Martinotti, E.; Saccomanni, G. Synthesis of 1,8-naphthyridine derivatives: Potential antihypertensive agents - Part VIII. Eur. J. Med. Chem. 1999, 34, 505-513.

14. Ferrarini, P.L.; Mori, C.; Badawneh, M.; Calderone, V.; Calzolari, L.; Loffredo, T.; Martinotti, E.; Saccomanni, G. Synthesis of 1,8-naphthyridine derivatives: Potential antihypertensive agents Part VII. Eur. J. Med. Chem. 1998, 33, 383-397.

15. Ferrarini, P.L.; Mori, C.; Badawneh, M.; Calderone, V.; Greco, R.; Manera, C.; Martinelli, A.; Nieri, P.; Saccomanni, G. Synthesis and $\beta$-blocking activity of (R,S)-(E)-oximeethers of 2,3dihydro-1,8-naphthyridine and 2,3-dihydrothiopyrano[2,3-b]pyridine: Potential antihypertensive agents - Part IX. Eur. J. Med. Chem. 2000, 35, 815.

16. Graf, H.; Franz, L.; Sauter, H.; Ammermann, E.; Pommer, E-H. Substituted 1,8-naphthyridine derivatives and fungicides containing them. U.S. Patent 4,801,592, 31 January 1989.

17. Saupe, T.; Schaefer, P.; Meyer, N.; Wuerzer, B.; Westphalen, K.O. Substituted 1,8naphthyridines, their preparation and their use as antidotes. U.S. Patent 5,258,356, 2 November 1993.

18. Cotrel, C.; Guyon, C.; Roussel, G.; Taurand, G. Anxiolytic amides derived from certain 1,8naphthyridine-2-amines. U.S. Patent 4,753,933, 28 June 1988.

19. Litvinov, V.P.; Roman, S.V.; Dyachenko, V.D. Naphthyridines. Structure, physicochemical properties and general methods of synthesis. Russ. Chem. Rev. 2000, 69, 201-220.

20. Smart, B.E. Fluorine substituent effects (on bioactivity). J. Fluorine Chem. 2001, 109, 3-11.

21. Druzhinin, S.V.; Balenkova, E.S.; Nenajdenko, V.G. Recent advances in the chemistry of $\alpha, \beta-$ unsatured trifluoromethylketones. Tetrahedron 2007, 63, 7753-7808.

22. Eichler, E.; Rooney, C.S.; Williams, H.W.R. 1,8-Naphthyridines. Part I. Synthesis of some trifluoromethyl-1,8-naphthyridines derivatives. J. Heterocycl. Chem. 1976, 13, 41-42.

23. Naik, T.R.R.; Naik, H.S.B.; Raghavendra, M.; Naik, S.G.K. Synthesis of thieno[2,3b] benzo[1,8]naphthyridine-2-carboxylic acids under microwave irradiation and interaction with DNA studies. ARKIVOC 2006, xv, 84-94.

24. Chen, S.; Chen, R.; He, M.; Pang, R.; Tan, Z.; Yang, M. Design, synthesis, and biological evaluation of novel quinoline derivatives as HIV-1 Tat-TAR interaction inhibitors. Bioorg. Med. Chem. 2009, 17, 1948-1956.

25. Pizzio, L.; Romanelli, G.; Vázquez, P.; Autino, J.; Blanco, M.; Cáceres, C. Keggin heteropolyacid-based catalysts for the preparation of substituted ethyl $\beta$-arylaminocrotonates, intermediates in the synthesis of 4-quinolones. Appl. Catal. A 2006, 308, 1531-1560.

26. Hauser, C.R.; Reynolds, G.A. Reactions of $\beta$-keto esters with aromatic amines. Syntheses of 2and 4-hydroxyquinoline derivatives. J. Am. Chem. Soc. 1948, 70, 2402-2404.

27. Manske, R.H.F.; Kulka, M. The Skraup synthesis of quinolines. Org. React. 1953, 7, 59. 
28. Bonacorso, H.G.; Righi, F.J.; Rodrigues, C.A.; Cechinel, C.A.; Costa, M.B.; Wastowski, A.D.; Martins, M.A.P.; Zanatta, N. New efficient approach for the synthesis of 2-alkyl(aryl)substituted 4H-pyrido[1,2-a]pyrimidin-4-ones. J. Heterocycl. Chem. 2006, 43, 229-233.

29. Brown, E.V. 1,8-Naphthyridines. I. Derivatives of 2- and 4-methyl-1,8-naphthyridines. J. Org. Chem. 1965, 30, 1607-1610.

30. Bonacorso, H.G.; Lourega, R.V.; Wastowski, A.D.; Flores, A.F.C.; Zanatta, N.; Martins, M.A.P. $\beta$-Alkoxyvinyl trichloromethyl ketones as $\mathrm{N}$-heterocyclic acylating agent. A new access to $5 \mathrm{H}$ thiazolo[3,2-a]pyrimidin-5-ones. Tetrahedron Lett. 2002, 43, 9315-9318.

31. Bonacorso, H.G.; Lourega, R.V.; Deon, E.D.; Zanatta, N.; Martins, M.A.P. The first synthesis of dihydro-3H-pyrido[2,3-b][1,4]diazepinols and a new alternative approach for diazepinone analogues. Tetrahedron Lett. 2007, 48, 4835-4838.

32. Bonacorso, H.G.; Lourega, R.V.; Righi, F.J.; Deon, E.D.; Zanatta, N.; Martins, M.A.P. Preparation of new 2-amino- and 2,3-diamino-pyridine trifluoroacetyl enamine derivatives and their application to the synthesis of trifluoromethyl-containing $3 H$-pyrido[2,3-b][1,4]diazepinols. J. Heterocycl. Chem. 2008, 45, 1679-1686.

33. Bonacorso, H.G.; Lourega, R.V.; Porte, L.M.F.; Deon, E.D.; Flores, A.F.C.; Zanatta, N.; Martins, M.A.P. Regiospecific synthesis of $3 H$-pyrido[2,3-b][1,4]diazepin-4(5H)-ones via haloform reaction with the isolation of $N^{3}$-[3-oxo-4,4,4-trichloroalk-1-en-1-yl]-2,3-diaminopyridine intermediates. J. Heterocycl. Chem. 2009, 46, 603-609.

34. Gerus, I.I.; Gorbunova, M.G.; Kukhar, V.P. $\beta$-Ethoxyvinyl polyfluoroalkyl ketones - versatile synthones in fluoroorganic chemistry. J. Fluorine Chem. 1994, 69, 195-198.

35. Bonacorso, H.G.; Duarte, S.H.G.; Zanatta, N.; Martins, M.A.P. Regiospecific Synthesis of 3alkyl-2-aryl-4-trifluoromethylbenzo[ $h]$ quinolines by intramolecular cyclization of $N$-(2-alkyl-1aryl-3-oxo-4,4,4-trifluorobut-1-en-1-yl)-1-naphthylamines. Synthesis 2002, 1037-1042.

36. Bonacorso, H.G.; Drekener, R.L.; Rodríguez, I.R.; Vezzosi, R.P.; Costa, M.B.; Martins, M.A.P.; Zanatta, N. Synthesis of new fluorine-containing dihydrobenzo[c]acridines from trifluoroacetyl dihydronaphthalene and substituted anilines. J. Fluorine Chem. 2005, 126, 1384-1389.

37. Bonacorso, H.G.; Moraes, T.S.; Zanatta, N.; Martins, M.A.P.; Flores, A.F.C. Synthesis of new trifluoromethyl-containing cycloalka[b]quinolines derived from alkoxycycloalkenes. ARKIVOC 2008, $x v i, 75-83$.

38. Bonacorso, H.G.; Moraes, T.S.; Zanatta, N.; Martins, M.A.P. Synthesis of new fluorinecontaining 1,2,3,4-tetrahydroacridines. Synth. Commun. 2009, 39, 3677-3686.

39. Bonacorso, H.G.; Bittencourt, S.R.T.; Lourega, R.V.; Flores, A.F.C.; Zanatta, N.; Martins, M.A.P. A Convenient synthetic method for fully conjugated 3-alkyl- and 3-aryl-5-trifluoromethyl-1methyl-1,2-thiazine 1-oxide from $\beta$-alkoxyvinyl trifluoromethyl ketones. Synthesis 2000, 1431-1434.

40. Bonacorso, H.G.; Wentz, A.P.; Bittencourt, S.T.R.; Marques, L.M.L.; Zanatta, N.; Martins, M.A.P. Synthesis of some $N$-[1-alkyl(aryl)-3-oxo-4,4,4-trichloro(trifluoro)-1-buten-1-yl]-oaminophenols and $o$-phenylenediamines as potential anticancer agents. Synth. Commum. 2002, 32, 335-341. 
41. Bonacorso, H.G.; Andrighetto, R.; Zanatta, N.; Martins, M.A.P. The unexpected cyclization routes of $N, N$ '-bis(oxotrifluoroalkenyl)-1,3-phenylenediamines in polyphosphoric acid medium. Tetrahedron Lett. 2010, 51, 3752-3755.

42. Effenberger, F.; Maier, R.; Schonwalder, K.H.; Ziegler, T. Enolether, XIII. Die acylierung von enolethern mit reaktiven carbonsäure-chloriden. Chem. Ber. 1982, 115, 2766-2782.

43. Kamitori, Y.; Hojo, M.; Masuda, R.; Fujitani, T.; Kobuchi, T.; Nishigaki, T. A new convenient synthetic method for 3-allyl-1,1,1-trifluoroacetylacetone and its derivatives. Synthesis 1986, 340-342.

44. Hojo, M.; Masuda, R.; Okada, E. A useful one-step synthesis of $\beta$-trihaloacetylvinyl ethers and trihaloacetylketene acetals. Synthesis 1986, 1013-1014.

45. Colla, A.; Martins, M.A.P.; Clar, G.; Krimmer, S.; Fischer, P. Trihaloacetylated enol ethers general synthetic procedure and heterocyclic ring closure reactions with hydroxylamine. Synthesis 1991, 483-486.

46. Ezell, E.L.; Thummel, R.P.; Martin, G.E. Correlation of resonances of strongly coupled spin systems via responses due to strong coupling in homonuclear two-dimensional $J$-resolved spectra: Total assignment of the ${ }^{1} \mathrm{H}-\mathrm{NMR}$ spectrum of 2-(2'-pyridyl)-1,8-naphthyridine. $J$. Heterocycl.Chem. 1984, 21, 817-823.

47. Flores, A.F.C.; Siqueira, G.M.; Freitag, R.; Zanatta, N.; Martins, M.A.P. Síntese de 2-trialoacetilcicloexanonas e -pentanonas: Um estudo comparativo dos rendimentos de reação de enoléteres, cetais e enaminas frente à trialometilacetilantes. Quim. Nova 1994, 17, 298-300.

48. Bonacorso, H.G.; Costa, M.B.; Moura, S.; Pizzuti, L.; Martins, M.A.P.; Zanatta, N.; Flores, A.F.C. Synthesis, ${ }^{17} \mathrm{O}$ NMR spectroscopy and structure of 2-trifluoroacetyl-1methoxycycloalkenes. J. Fluorine Chem. 2005, 126, 1396-1402.

Sample Availability: Samples of the compounds $\mathbf{2 b}, \mathbf{2 k}, \mathbf{3 a - m}, \mathbf{4 c}, \mathbf{4 f}, \mathbf{4 g}, \mathbf{4 i}, \mathbf{4 j}$ are available from the authors.

(C) 2011 by the authors; licensee MDPI, Basel, Switzerland. This article is an open access article distributed under the terms and conditions of the Creative Commons Attribution license (http://creativecommons.org/licenses/by/3.0/). 